\title{
MORFOMETRIA COMPARADA DE CTENOSCLAENA GRACILICIRRḦUS, PARALONCHURUS BRASILIENSIS E MICROPOGONIAS FURNIERI (TELEOSTEI: SCIAENIDAE) PELA ANÁLISE MULTIVARIADA DE REDES DE TRELIÇAS
}

\author{
Mauro José Cavalcanti ${ }^{1}$ \\ Paulo Roberto Duarte Lopes ${ }^{2}$
}

\begin{abstract}
Comparative morphometrics of Ctenosciaena gracilicirrhus, Paralonchurus brasiliensis and Micropogonias furnieri (Teleostei: Sciaenidae) by multivariate analysis of truss networks. Morphological differentiation among three species of sciaenid fishes, Ctenosciaena gracilicirrhus, Paralonchurus brasiliensis and Micropogonias fumieri, was analyzed by multivariate statistical techniques, using measuraments obtained from truss networks based on anatomical landmarks. Principal components analysis of interlandmark distances defined by the truss system showed that the individuals of Ctenosciaena gracilicirrhus are different from those of the other two species in relation to shape, being more similar to the individuals of Micropogonias furnieri in relation to size.
\end{abstract}

\section{INTRODUÇÃO}

O estudo por métodos numéricos da forma corporal em relação ao tamanho, denominado morfometria, tem-se mostrado de grande importância na compreensão da biologia evolutiva dos organismos (Blackith \& Reyment, 1971). Os peixes, cujas formas são geralmente bem definidas em função do hidrodinamismo, facilitando a quantificação, têm sido objeto de muitos estudos morfométricos.

A maioria dos estudos morfométricos recentes faz uso de técnicas de análise estatística multivariada, que permitem expressar padrões de variação e covariação de caracteres em um número reduzido de dimensões, fornecendo meios de visualizar graficamente e comparar esses padrões entre diferentes grupos de organimos (Blackith \& Reyment, 1971).

Contudo, tais estudos são, via de regra, efetuados sobre um conjunto relativamente reduzido de variáveis morfométricas, selecionadas sem qualquer critério objetivo, o que torna imprecisas as diferenças de formas porventura detectadas e impede ou dificulta a discriminação de grupos de organismos com base nessas diferenças (Humphries et al., 1981).

Strauss \& Bookstein (1982) sugeriram um protocolo geométrico preciso para a quantificação da forma de organismos, notadamente peixes, denominado "redes de treliças" (truss networks), o qual possibilita a realização de medidas a partir da associação de pontos anatômicos (marcos) definidos com base na morfologia externa, pressupondo-se

1. Instituto de Biologia, Universidade Federal do Rio de Janeiro, 21949, Rio de Janeiro, RJ.

2. Departamento de Ciências Biológicas, Universidade Estadual de Feira de Santana, 44061, Feira de Santana, BA. 
a homologia dos mesmos entre as formas sendo comparadas. Somente com base nesta suposição é que as medidas morfométricas podem revestir-se de um significado biológico definido e interpretável, permitindo que comparações válidas entre diferentes organismos sejam feitas (Humphries et al., 1981; Strauss \& Bookstein, 1982).

O método das redes de treliças permite: (1) detectar sistematicamente diferenças de forma em todas as direções, possibilitando cobrir toda a configuração de marcos anatômicos dentro do contorno do organismo; (2) reconstruir a forma corporal do organismo a partir da configuração inicial de marcos anatômicos; (3) verificar e compensar erros aleatórios de medição; (4) computar e traçar "formas médias" com base nas formas de uma amostra de indivíduos; (5) descrever padrões multidimensionais de crescimento e alometria dentro de populações; (6) discriminar entre os grupos de organismos que variam em forma e tamanho (Strauss \& Bookstein, 1982).

Recentemente, vários estudos morfométricos de peixes, tanto marinhos quanto de água doce, têm sido realizados por meio desta metodologia (Strauss \& Fuiman, 1985; Shao \& Chang, 1985; Rauchenberger, 1988). O objetivo deste trabalho é ilustrar o uso da metodologia das redes de treliças em conjunto com as técnicas de morfometria multivariada, analisando os padrões de diferenciação morfológica entre três espécies de peixes ocorrentes no Atlântico ocidental, da família Sciaenidae (Ctenosciaena gracilicirrhus Metzelaar, 1919, Paralonchurus brasiliensis Steindachner, 1875 e Micropogonias furnieri Desmarest, 1823).

\section{MATERIAL E MÉTODOS}

Os exemplares de Ctenosciaena gracilicirrhus, Paralonchurus brasiliensis e Micropogonias furmieri utilizados para a análise encontram-se depositados na coleção do Laboratório de Ictiologia do Departamento de Zoologia do Instituto de Biologia da Universidade Federal do Rio de Janeiro. A maior parte do material examinado é proveniente de coletas efetuadas ao longo do litoral do estado do Rio de Janeiro, Brasil.

Foram utilizados um total de 54 individuos (sendo 17 de C. gracilicirrhus, 47,0$118,0 \mathrm{~mm}$ de comprimento padrão (CP), 23 de $P$. brasiliensis, $115,0-184,0 \mathrm{~mm}(\mathrm{CP}) \mathrm{e}$ 14 de $M$. furnieri, 98,0-280,0 mm (CP), tomando-se 21 medidas morfométricas de cada exemplar, a partir do protocolo geométrico de redes de treliças (Strauss \& Bookstein, 1982; Strauss \& Fuiman, 1985). As medidas foram obtidas com o auxilio de um paquímetro, com precisão de $0,05 \mathrm{~mm}$. Os marcos selecionados foram: 1) Margem anterior da maxila superior; 2) Região interorbital; 3) Base do espinho da nadadeira pélvica;4) Base do primeiro espinho da primeira nadadeira dorsal; 5) Margem anterior do ânus; 6) Base do último espinho da primeira nadadeira dorsal; 7) Base do último raio da nadadeira anal; 8) Base do último raio da segunda nadadeira dorsal; 9) Margem inferior do pedúnculo caudal; 10) Margem superior do pedúnculo caudal (Figura 1).

Para a execução da análise estatística, as medidas foram transformadas em logarítmos, a fim de normalizar sua distribuição e homogeneizar as variâncias (Sokal \& Rohlf, 1981). Esta transformação mostra-se apropriada na comparação de formas que apresentam crescimento alométrico, além de facilitar a análise estatística dos dados e permitir a aplicação do modelo de alometria multivariada de Jolicoeur (Jolicoeur, 1963).

A análise morfométrica foi baseada na ordenação pelos componentes principais (Morrison, 1976), uma das técnicas multivariadas mais conhecidas e utilizadas em bio- 
logia evolutiva e taxonomia (Blackith \& Reyment, 1971; Sneath \& Sokal, 1973; Reis, Cunha, Garavello \& Abe, 1987). Os autovetores e seus autovalores associados foram obtidos a partir da matriz de covariância dos dados logaritmizados, permitindo representar em um mínimo de fatores a maior parte da variação dos caracteres originais e possibilitando avaliar as relações entre as três espécies, estabelecidas por sua proximidade no espaço delimitado pelos componentes.

Além disto, calculou-se o coeficiente de correlação vetorial (Strauss \& Fulman, 1985; Morrison, 1976) entre os coeficientes do primeiro componente principal, a fim de testar a premissa básica de direções de variação semelhante nas três espécies estudadas, implícita na análise de componentes principais, quando a amostra contêm grupos provenientes de populações estatísticas diferentes. Estes coeficientes foram calculados a partir do produto interno dos coeficientes do primeiro componente principal, computado separadamente para cada espécie, indicando direções de variação iguais quando seus valores aproximam-se de 1 e direções diferentes quando seus valores são próximos de 0 .

A técnica de análise de componentes principais foi também utilizada para testar a ocorrência de um padrão alométrico multivariado nestas três espécies, por meio de estimativas dos coeficientes alométricos (Jolicoeur, 1963; Strauss, 1985) das variáveis mor-

fométricas obtidas da rede de treliças. Estes coeficientes foram calculados escalonandose os coeficientes das variáveis no primeiro componente principal, de modo a tomar sua soma dos quadrados igual a 1, podendo ser interpretados como coeficientes de crescimento relativo (Rauchenberger, 1988). Os valores dos coeficientes alométricos maiores do que 1 descrevem alometria positiva em relação ao tamanho, enquanto que os valores menores que 1 indicam alometria negativa (Jolicoeur, 1963). Assim, as variáveis morfométricas alometricamente positivas crescem relativamente mais rápido que a média, contribuindo mais fortemente para o fator do tamanho geral representado pelo primeiro componente principal. Coeficientes alométricos foram também calculados separadamente para cada uma das três espécies, a fim de detectar diferenças nos padrões de crescimento relativo entre as mesmas.

Para o processamento dos dados, foi utilizado um microcomputador da linha IBM-PC do Laboratório de Informática do CCS-UFRJ, sendo a ordenação pelos componentes principais efetuada através do programa PCA, adaptado de Wahlstedt \& Davis (1968).

\section{RESULTADOS}

Os valores dos coeficientes de correlação vetorial apresentaram-se muito semelhantes entre as três espécies $(>0.97)$, indicando assim direções de variação iguais nestas espécies.

Os três primeiros componentes principais deram conta de $96,2 \%$ da variação total presente na matriz de covariância dos 21 caracteres morfométricos obtidos da rede de treliças na amostra combinada das três espécies. $O$ primeiro componente principal explicou cerca de $92 \%$ da variação total, podendo ser ińterpretado como um fator de tamanho geral, uma vez que todas as variáveis morfométricas apresentaram coeficientes positivos e correlações positivas $(\mathrm{P}<0.05)$ com este componente (Tabela 1). Contudo, a grande amplitude dos coeficientes do primeiro componente (0.13-0.28), indica que nem todos os caracteres morfométricos obtidos da rede de treliças contribuiram igualmente 
para este fator de tamanho geral, isto é, que os vários caracteres contribuíram com alometrias diferentes para a forma nestas três espécies. Isto pode ser igualmente constatado pelos valores dos coeficientes alométricos calculados a partir dos coeficientes do primeiro componente principal (Tabela 1), verificando-se que alguns dos caracteres morfométricos obtidos da rede de treliças apresentam uma forte alometria positiva, e outros mostram alometrias negativas de diferentes intensidades, em relação ao fator de tamanho geral representado pelo primeiro componente principal; por outro lado, um dos caracteres (a distância entre os marcos anatômicos 5 e 6; Figura 1 e Tabela 1) é aproximadamente isométrico. Os coeficientes alométricos para cada espécie são apresentados na Figura 4, indicados diretamente sobre os diagramas da rede. O segundo componente principal explicou cerca de $2,86 \%$ da variação e o terceiro cerca de $1,36 \%$; o restante da variabilidade ficou distribuída entre os demais 18 componentes. $\mathrm{O}$ segundo e o terceiro componentes principais apresentaram coeficientes positivos e negativos (Tabela 1), contendo informação referente à forma nas três espécies analisadas.

$\mathrm{Na}$ projeção dos escores os indivíduos de Micropogonias, Paralonchurus e Ctenosciaena, nota-se uma dependência das projeções dos escores do segundo componente em relação ao primeiro (Figura 2), a qual é provavelmente devida à alometria. Assim, um aumento no tamanho geral tende a acarretar uma alteração na forma destas espécies durante o seu crescimento. Pode-se notar que os indivíduos maiores de Ctenosciaena superpõem-se aos menores de Micropogonias no espaço dos dois primeiros componentes principais (Figura 2); ainda assim, a separação bastante nítida observada entre as três espécies não tornou necessária a aplicação do método da distorção (shear) (Humphries et al., 1981; Reis, Cunha, Garavello e Abe, 1987) ao primeiro componente principal.

O gráfico dos escores dos individuos de Micropogonias, Paralonchurus e Ctenosciaena nos segundo e terceiro componentes principais mostra, por sua vez, que C. gracilicirrhus diferencia-se das outras duas espécies no que diz respeito à forma (Figura 3), superpondo-se, neste caso, os individuos de $M$. furmieri e $P$. brasiliensis. Isto é confirmado pela análise de cada espécie separadamente, onde os valores relativamente mais altos do segundo componente principal na região mediana mostram que $C$. gracilicirrhus possui um corpo mais alto que $P$. brasiliensis e $M$. furnieri, às quais apresentam, por sua vez, pedúnculos caudais mais alongados.

\section{CONCLUSÕES}

É importante notar que este trabalho é um ensaio de avaliação metodológica, não pretendendo-se que seus resultados ou conclusões sejam extensivos a outras espécies dos gêneros aqui analisados ou aos demais gêneros da família Sciaenidae. Seus resultados indicam que a metodologia das redes de treliças, combinada às técnicas de análise estatística multivariada, revela um grande potencial na investigação dos padrões de diferenciação morfológica e de crescimento em peixes, como já tem sido demonstrado na literatura recente (Strauss \& Bookstein, 1982; Strauss \& Fuiman, 1985; Shao \& Chang, 1985; Rauchenberger, 1988). Deve-se ressaltar ainda sua importância nos estudos de taxonomia e sistemática, podendo inclusive ser utilizada para diferenciar espécies congêneres (Strauss \& Bookstein, 1982; Rauchenberger, 1988), bem como no estabelecimento de hipóteses sobre relações filogenéticas entre espécies (Shao \& Chang, 1985). A maior precisão fornecida pela técnica das redes de treliças torna fortemente recomendável sua utilização em estudos comparativos da forma de organismos. 
Vol. 7(4), 1990

\section{TABELA 1}

Coeficiente (CPI, CPII e CPIII) da análise de componentes principais dos 21 caracteres morfométricos das amostras combinadas de Micropogonias, Paralonchurus e Ctenosciaena. r e k são, respectivamente, o coeficiente de correlação de cada caráter com o primeiro componente principal e o coeficiente de alometria multivariada de cada caráter.

$\begin{array}{lccccc}\text { Caracteres } & \text { CPI } & \text { CPII } & \text { CPIII } & \text { r } & \text { k } \\ & & & & & \\ \text { 1. D1-2 } & 0.1892 & -0.2644 & 0.3326 & 0.92 & 0.88 \\ \text { 2. D1-3 } & 0.2274 & -0.1240 & 0.1152 & 0.98 & 1.06 \\ \text { 3. D1-4 } & 0.2028 & -0.1703 & 0.1431 & 0.98 & 0.94 \\ \text { 4. D2-3 } & 0.2127 & -0.1116 & 0.0830 & 0.99 & 0.99 \\ \text { 5. D2-4 } & 0.2038 & -0.1174 & 0.0159 & 0.98 & 0.95 \\ \text { 6. D3-4 } & 0.1972 & -0.2454 & 0.0698 & 0.97 & 0.92 \\ \text { 7. D3-5 } & 0.2539 & -0.1499 & -0.1392 & 0.97 & 1.18 \\ \text { 8. D3-6 } & 0.1947 & -0.2025 & 0.1188 & 0.97 & 0.90 \\ \text { 9. D4-5 } & 0.2319 & -0.0088 & -0.0705 & 0.99 & 1.08 \\ \text { 10. D4-6 } & 0.1787 & -0.0969 & -0.0344 & 0.96 & 0.83 \\ \text { 11. D5-6 } & 0.2185 & -0.0305 & -0.0308 & 0.98 & 1.01 \\ \text { 12. D5-7 } & 0.1710 & -0.1253 & 0.4680 & 0.91 & 0.79 \\ \text { 13. D5-8 } & 0.2352 & 0.1350 & 0.2553 & 0.96 & 1.09 \\ \text { 14. D6-7 } & 0.2456 & 0.0759 & -0.0136 & 0.98 & 1.14 \\ \text { 15. D6-8 } & 0.2854 & 0.3233 & 0.0438 & 0.96 & 1.32 \\ \text { 16. D7-8 } & 0.2574 & 0.2804 & -0.0350 & 0.97 & 1.20 \\ \text { 17. D7-9 } & 0.2640 & 0.3445 & -0.2866 & 0.94 & 1.23 \\ \text { 18. D7-10 } & 0.2326 & 0.2592 & -0.1582 & 0.97 & 1.08 \\ \text { 19. D8-9 } & 0.1836 & -0.1654 & -0.1748 & 0.94 & 0.85 \\ \text { 20. D8-10 } & 0.1314 & -0.5367 & -0.5991 & 0.72 & 0.61 \\ \text { 21. D9-10 } & 0.2061 & -0.0367 & -0.1373 & 0.98 & 0.96\end{array}$

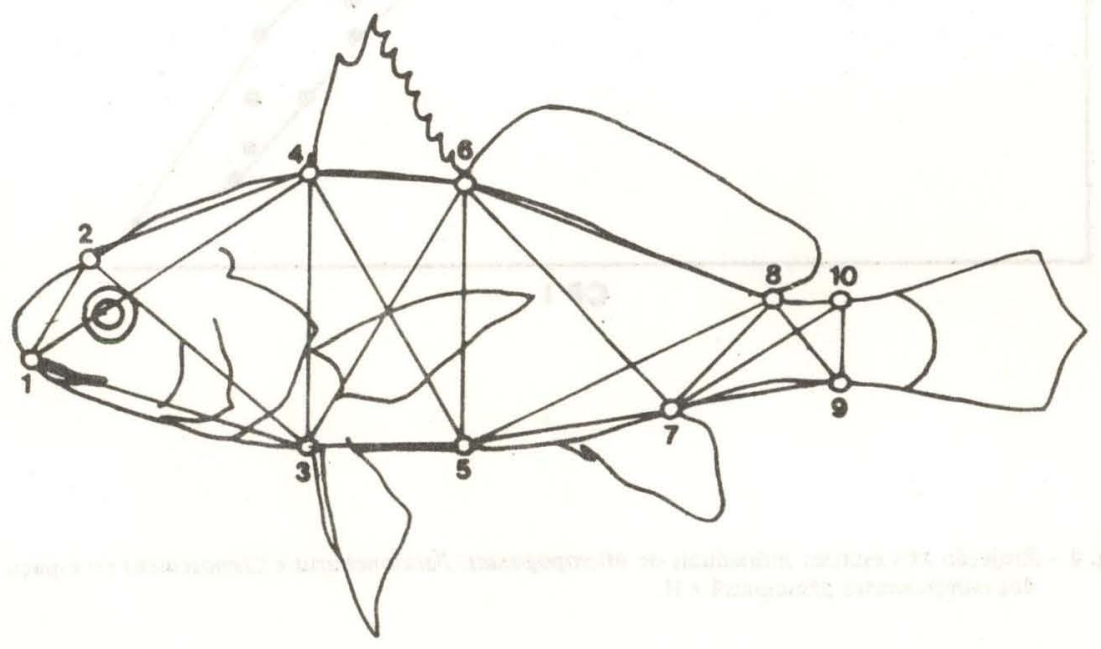

Fig. 1 - Diagrama da rede de treliças para Micropogonias furnieri (linhas tracejadas) obtida a partir dos marcos anatômicos (numerados). 
Revta bras. Zool.

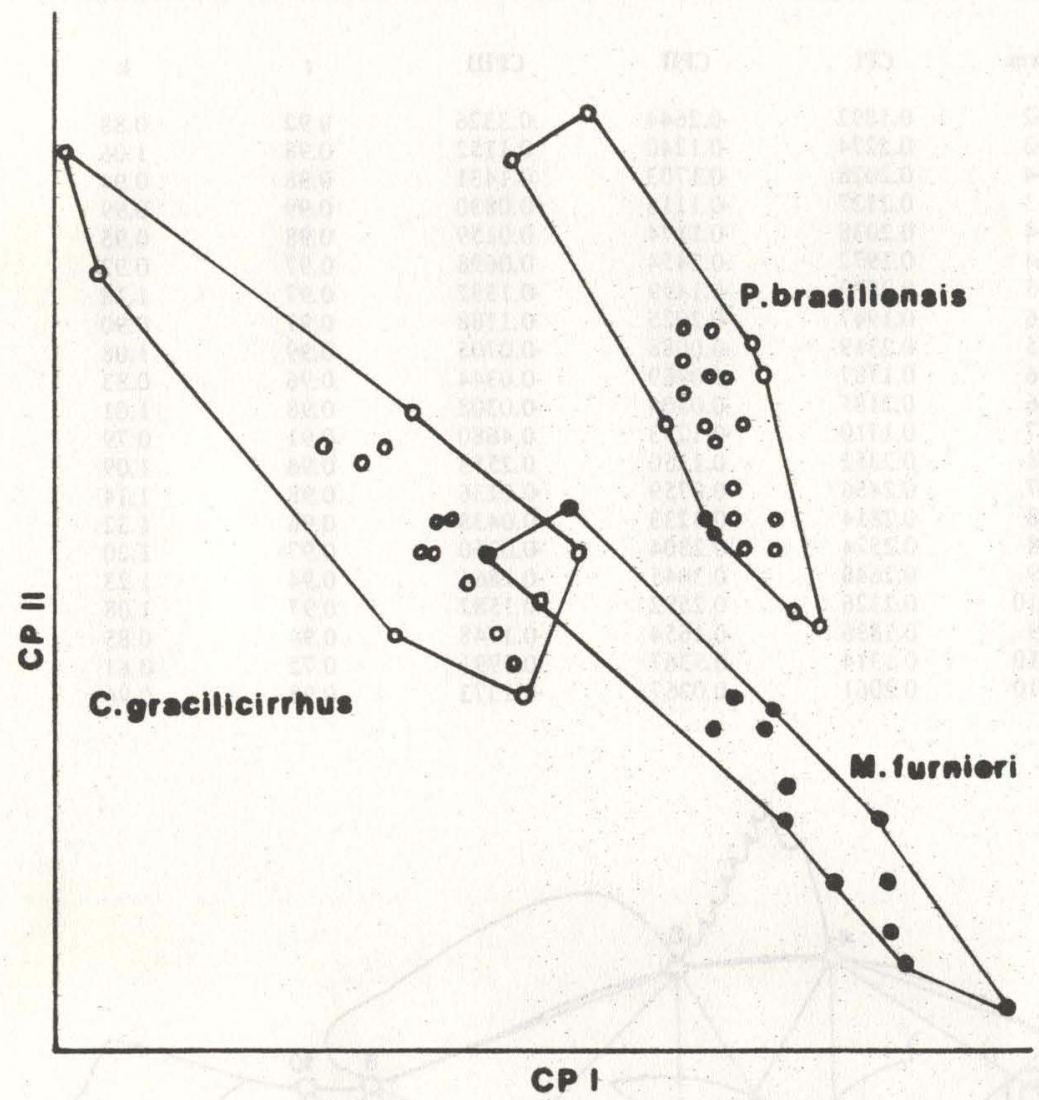

Fig. 2 - Projeção dos escores individuais de Micropogonais, Paralonchurus e Ctenosciaena no espaço dos componentes principais I e II. 
Vol. 7(4), 1990

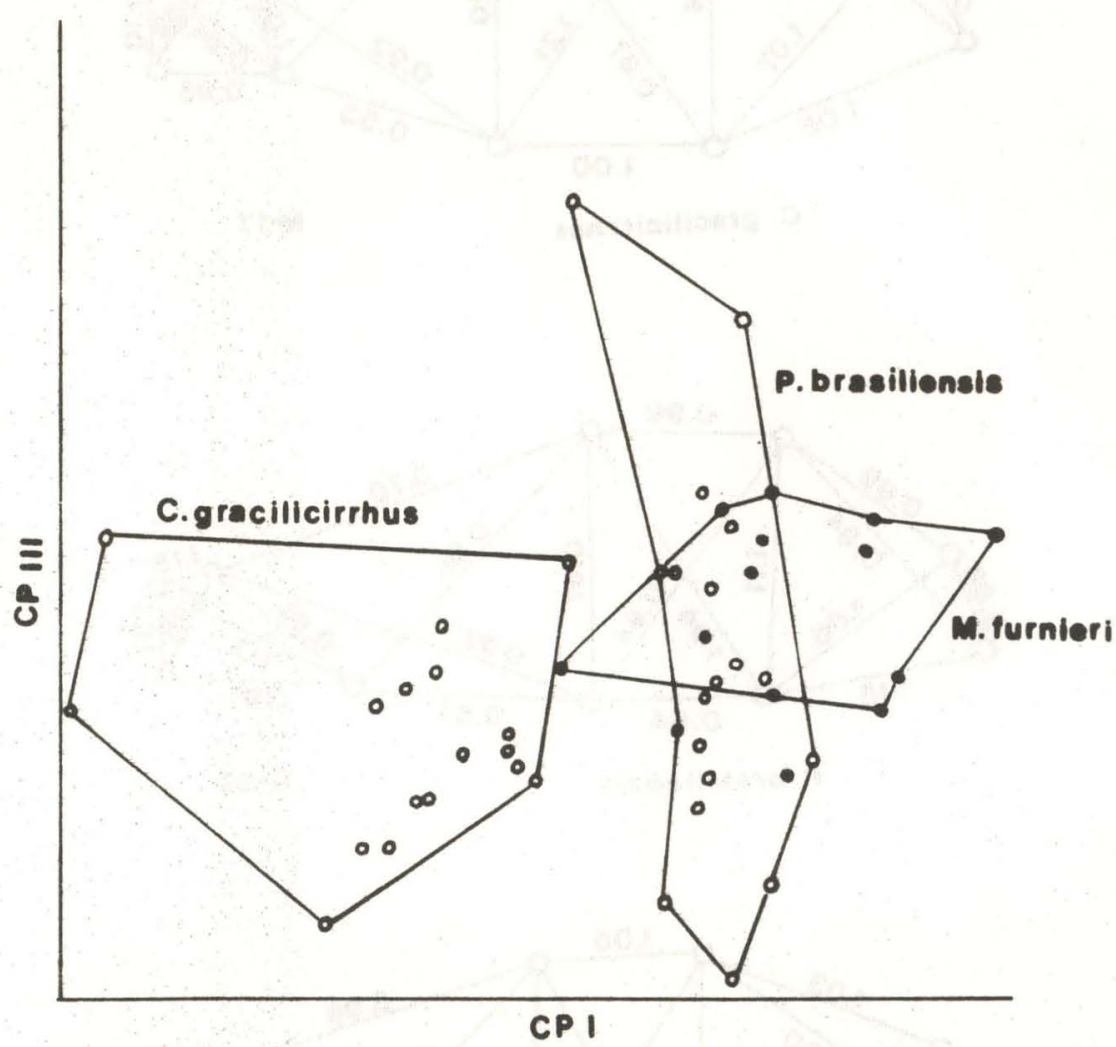

Fig. 3 - Projeção dos escores individuais de Micropogonias, Paralonchunus e Ctenosciaena no espaço dos componentes principais I e III. 
Revta bras. Zool.

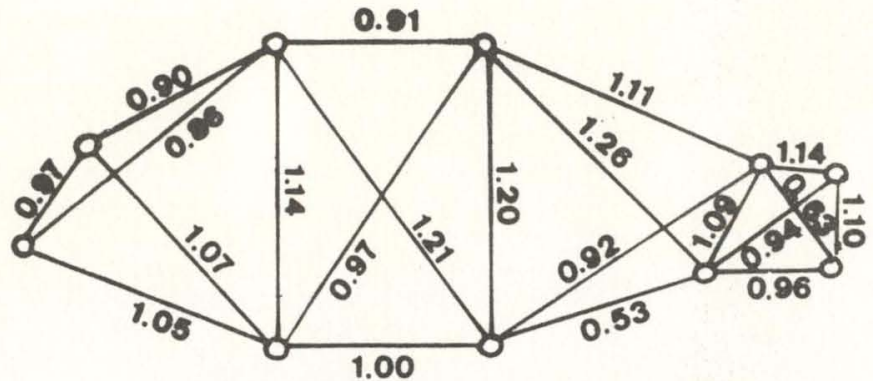

C. gracilicirrhus

$N=17$

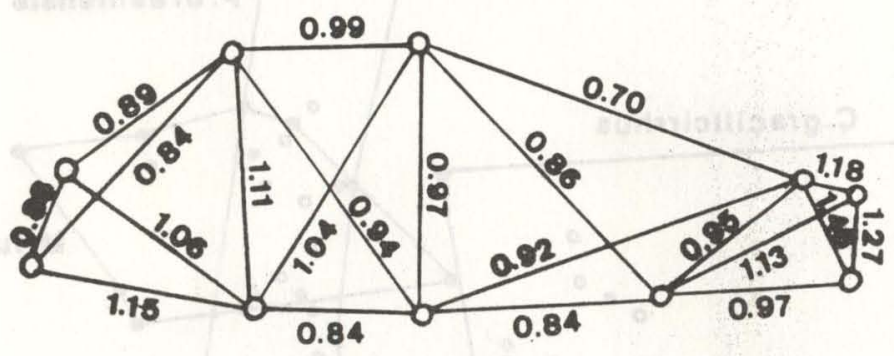

P. brasiliensis

$N=23$

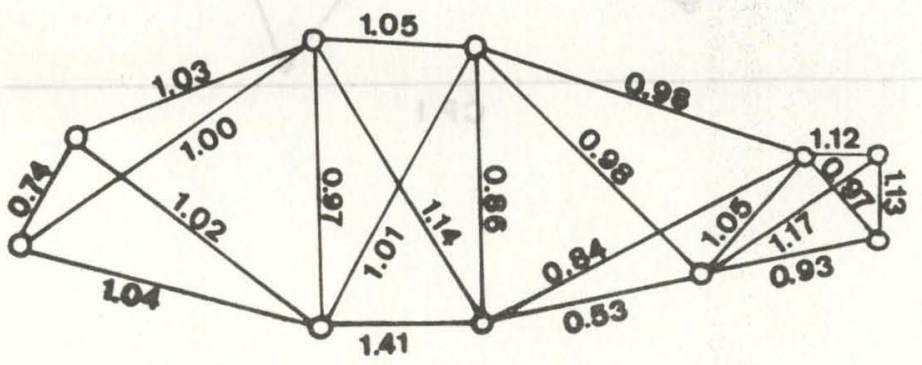

M. furnieri

$N=14$

Fig. 4 - Coeficientes alométricos multivariados para cada espécie, indicados para cada segmento da rede de treliças. 


\section{AGRADECIMENTOS}

Ao Dr. Sérgio Furtado dos Reis (Departamento de Parasitologia - Universidade Estadual de Campinas) pela orientação e valiosas sugestões.

Ao Dr. George John Shepherd (Departamento de Botânica - Universidade Estadual de Campinas) pela doação do programa PCA utilizado neste estudo.

Ao Prof. Mario Jorge Ignacio Brum (Departamento de Zoologia - Universidade Federal do Rio de Janeiro) pela permissão de utilizar o material depositado na coleção ictiológica desta instituição. cedidas.

$\grave{A}$ equipe do Laboratório de Informática do CCS-UFRJ, pelas facilidades con-

\section{REFERÊNCIAS BIBLIOGRĀFICAS}

BLACKITH, R.E. \& REYMENT, R.A., 1971. Multivariate Morphometrics. Academic Press, London, $410 \mathrm{p}$.

HUMPHRIES et al., 1981. Multivariate discrimination by shape in relation to size. Syst. Zool. 30: 291-308.

JOLICOEUR, P., 1963. The multivariate generalization of the allometry equation. Biometrics 19: $497-499$.

MORRISON, D.F., 1976. Multivariate Statistical Methods. McGraw-Hill, New York, 415 p.

RAUCHENBERGER, M., 1988. A new species of Allodontichthys (Cyprinodontiformes: Goodeidae), with comparative morphometrics for the genus. Copeia 1988:433-441.

REIS, S.F., CUNHA, R.A., GARAVELLO, J.C. \& ABE, A.S., 1987. Discriminação pela forma em relação ao tamanho: um exemplo com peixes do gênero Leporinus. Ciênc. Cult. 39:757-761.

SHAO, K.T. \& CHANG, W., 1985. Phylogenetic inference of Priacanthidae from morphometric studies. Bull. Inst. Zool., Academia Sinica 24: 273-288.

SNEATH, P.H.A. \& SOKAL, R.R., 1973. Numerical Taxonomy: The Principles and Practice of Numerical Classification. W.H. Freeman, San Francisco, 573 p.

SOKAL, R.R. \& ROHLF, F.J., 1981. Biometry: The Principles and Practice of Statistics in Biological Research. W.H. Freeman, San Francisco, 859 p.

STRAUSS, R.E., 1985. Evolutionary allometry and variation in body form in the South American catfish genus Corydoras (Callichthyidae). Syst. Zool. 34:381-396

STRAUSS, R.E. \& BOOKSTEIN, F.L., 1982. The truss: body form reconstructions in morphometrics. Syst. Zool. 31:113-135.

STRAUSS, R.E. \& FUIMAN, L.A., 1985. Quantitative comparisons of body form and allometry in larval and adult Pacific sculpins (Teleostei: Cottidae). Can. J. Zool. 63:1582-1589.

WAHLSTEDT, W.J. \& DAVIS, J.C., 1968. FORTRAN IV program for computation and display of principal components. Kansas Geol. Surv. Computer Contrib. (21), 27 p. 